\title{
A smart algorithm for fault detection and location in electric power distribution system
}

\author{
Hamid Touijer', Mohammed El Alami², Mustapha Zahri ${ }^{3}$, Mohamed Habibi ${ }^{4}$ \\ ${ }_{1,2,4}$ Laboratory of Electronic Systems, Information Processing, Mechanics and Energetics, Faculty of Science, Ibn Tofail \\ University, Morocco \\ ${ }^{3}$ General Manager of the Electricity and Water Distribution Company in El Jadida, Morocco
}

\begin{tabular}{l} 
Article Info \\
\hline Article history: \\
Received Mar 30, 2021 \\
Revised Sep 28, 2021 \\
Accepted Aug 7, 2021 \\
\hline
\end{tabular}

Keywords:

Electrical power distribution system

Fault location

New algorithm

\begin{abstract}
In an electric power distribution system (EPDS), fault location accuracy is critical for system stability. In the past, several algorithms have shown that they are inefficient. However, the results of these algorithms have been shown to be inefficient, and they should not be used for every sort of the faults. This paper presents a new algorithm capable to determine the location of fault accurately with low error rate. It is based on the voltage and current calculation at the source station for different types of faults by using either one power supply or double power supply. The work includes the formulation analytical development as simulation test results. The test results are produced by numerical simulation using data from a recognized distribution line in the literature.
\end{abstract}

This is an open access article under the $\underline{C C B Y-S A}$ license.

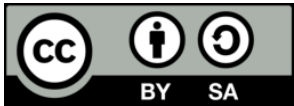

\section{Corresponding Author:}

Hamid Touijer

Laboratory of Electronic Systems, Information Processing, Mechanics and Energetics

Faculty of Science, Ibn Tofail University, Morocco

Email: hamid.touijer@gmail.com

\section{INTRODUCTION}

Fault location estimation is an important aspect in power systems restoration. This estimate helps to reduce the time without the injection renewable energy in EPDS to locate the defect therefore improves power quality indexes. The purpose of this work is to ensure this energy while avoiding the faults that may hinder its flow. Based on such concern, several methods have been proposed for the precision of the location [1]-[6] and fault detection by using Beaglebone black card [7] in real-time.

Various fault location methods were developed in the past. For example, Novosel et al. [8], use the apparent impedance which is defined as the ratio of the selected voltage to the current over the type of fault and the faulty phases, to locate faults. In Das [9], locates the sections and then the distance to the fault in this calculated section. Yang and Springs [10] propose a defect localization method that corrects the effects of defect resistance. The method proposed by Das et al. [11] used fundamental frequency voltages and currents measured at a line terminal before and during failure. In the method proposed by Saha et al. [12] estimates the location of the fault by comparing the design impedance of the power supply assuming faults of each section line. Choi et al. [13] locate the faults by solving a quadratic equation resulting from the direct circuit analysis. In Senger et al. [14] proposed a method based on measurement provided by intelligent electronic devices (IED). In Kim, et al. [15] the estimated location of outages on distribution power supplies using power quality monitoring data. A way to optimally place faulty circuit indicators along the feeder is developed by Almeida et al. [16]. Methods of reducing and eliminating uncertainty about the location of the fault are elaborated by Krishnathevar and Ngu [17], as well Avendano-Mora et al. [17], [18]. In Sadeh et al. 
[19] suggested a new algorithm for radial distribution systems using modal analysis. Wanjing et al. [20] presented a new method based on two types of fault location approaches using the line to neutral or the line to measure from the line to the substation in. Khoudry et al. [21] proposed a novel technique based on machine learning algorithms. In Abbas et al. [22] proposed a method based on Single line to ground fault detection and location in medium voltage distribution system network based on neural network. In [23] Charalampos et al. [23] a voltage-based fault location algorithm for medium voltage active distribution systems.

These proposed methods that we have highlighted above have the weaknesses in precision which make them not effective because they require algorithms adopted for each type of fault. The goal of this work is to develop a new algorithm capable to valid and minimize the error of all types of faults without. The structure of the paper is arranged as follows: The new single line to ground fault location formulation is described in Section 2. The Fault Location methodology is shown in Section 3. The test results and comparison are presented in Section 4 and the conclusions are presented in Section 5.

\section{MATHEMATICAL FAULT LOCATION FORMULATION}

To properly represent the fault location portion of the electrical network, we begin with a model diagram of a network during a fault, as shown in Figure 1.

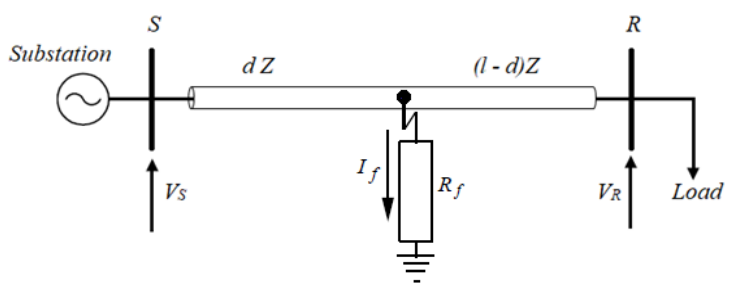

Figure 1. Model of a single-phase distribution line

$\mathrm{Z}$ : Characteristic line impedance

$\mathrm{V}_{\mathrm{s}}$ : Voltage at the source

$\mathrm{V}_{\mathrm{R}}$ : Voltage at the load

$\mathrm{V}_{\mathrm{F}}$ : Fault voltage

$\mathrm{I}_{\mathrm{S}} \quad$ : Current at the source

$I_{R} \quad$ : Current at the load

$\mathrm{I}_{\mathrm{F}} \quad$ : Fault current

1 : The row total length

d : The default distance from the source

From the electrical diagram we presented in Figure 1 we found (1).

$$
V_{s}=d Z I_{s}+V_{f}
$$

The complex notation is:

$$
\begin{aligned}
& V_{s}=V_{s}^{r}+j V_{s}^{i} \\
& I_{s}=I_{s}^{r}+j I_{s}^{i} \\
& V_{f}=V_{f}^{r}+j V_{f}^{i} \\
& I_{f}=I_{f}^{r}+j I_{f}^{i}
\end{aligned}
$$

In the simplest layered parameter model of an overhead line, series resistance (RL) and reaction (XL) are included only Figure 2. Such a model is considered as Figure 2 to represent a short line which is generally less than $80 \mathrm{~km}$ long [24]. 


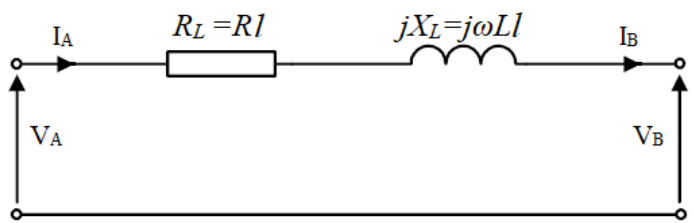

Figure 2. Line model

$R, L \quad: \quad$ Line resistance and inductance per unit length

$\omega$ : Angular fundamental frequency

$l \quad: \quad$ Line length

So, the line impedance is:

$$
Z=R_{L}+j X_{L}
$$

We replace this impedance in (1).

$$
V_{s}^{r}+j V_{s}^{i}=d\left(R_{L}+j X_{L}\right)\left(I_{s}^{r}+j I_{s}^{i}\right)+R_{f}\left(I_{f}^{r}+j I_{f}^{i}\right)
$$

We find (7)

$$
V_{s}^{r}+j V_{s}^{i}=\left[d\left(R_{L} I_{s}^{r}-X_{L} I_{s}^{i}\right)+R_{f} I_{f}^{r}\right]+j\left[d\left(R_{L} I_{s}^{i}+X_{L} I_{s}^{r}\right)+R_{f} I_{f}^{i}\right]
$$

Take,

$$
A=\left(R_{L} I_{s}^{r}-X_{L} I_{S}^{i}\right) \text { And } B=\left(R_{L} I_{s}^{i}+X_{L} I_{S}^{r}\right)
$$

So, we find the following system of:

$$
\begin{gathered}
\left(\begin{array}{l}
V_{s}^{r} \\
V_{s}^{i}
\end{array}\right)=\left(\begin{array}{ll}
A & I_{f}^{r} \\
B & I_{f}^{i}
\end{array}\right)\left(\begin{array}{l}
d \\
R_{f}
\end{array}\right) \\
\left\{\begin{array}{l}
V_{s}^{r}=d A+R_{f} I_{f}^{r} \\
V_{s}^{i}=d B+R_{f} I_{f}^{i}
\end{array}\right.
\end{gathered}
$$

Therefore

$$
d=\frac{\left(V_{s}^{r} I_{f}^{i}-V_{s}^{i} I_{f}^{r}\right)}{\left(A I_{f}^{i}-B I_{f}^{r}\right)}
$$

We have from the diagram in Figure 1.

$$
I_{s}=I_{f}+I_{R}
$$

At the fault

$$
I_{R}<<I_{f}
$$

Therefore

$$
I_{s}=I_{f}
$$

So, (12) becomes:

$$
d=\frac{\left(V_{S}^{r} I_{S}^{i}-V_{S}^{i} I_{S}^{r}\right)}{\left(A I_{S}^{i}-B I_{S}^{r}\right)}
$$


Based on this development as well as mathematical evidence, we have found a new, more efficient algorithm for cable fault accuracy.

\section{FAULT LOCATION ALGORITHM}

The algorithm procedure starts after the fault detection. There are $\mathrm{n}$ branches in the system. The following method for detecting the problematic site is begun for each branch:

- Voltage and current measurement at the source

- Comparing the voltage with a voltage referance (Vref)

- While the fault is detected the charging current is low, due to the voltage drops and the dynamics of the systems during the blackout. Hence, the source current during the fault is the same as the current source as show in the (14) and (16).

- The fault distance is calculated by (17).

The fault location algorithm is displayed in Figure 3.

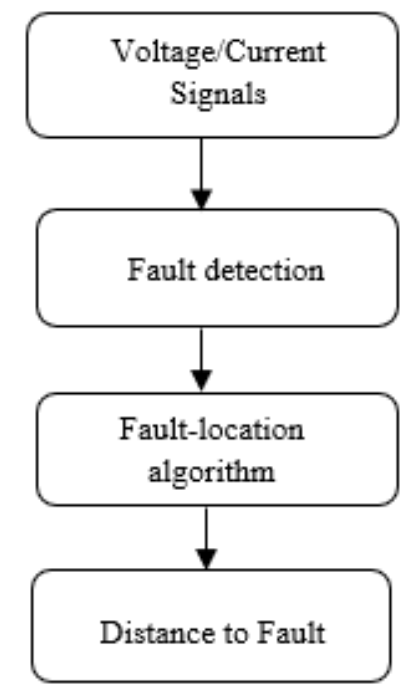

Figure 3. A new fault location algorithm

\section{RESULT AND COMPARISON}

The system studied is illustrated in Figure 4 for the two cases, the first with a single power supply in Figure 4 (a) and the second with a double power supply Figure 4 (b). It is part of the distribution network underground. The maximum length between two distribution stations in our city is $6 \mathrm{~km}$ and the voltage is 20 KV. It was simulated using a distributed parameter line model as shown in Table 1. Using MATLAB [25] as simulation tool. The proposed technique is extensively tested to verify its efficiency and accuracy. The performance of fault location algorithms is generally measured by the errors over the total line distance:

$$
\text { err }=\left|\frac{d_{\text {real }}-d_{\text {estimer }}}{l_{\text {total }}}\right|
$$

$$
\begin{aligned}
& d_{\text {real }}: \text { The actual fault distance. } \\
& d_{\text {estimer }}: \text { The distance obtained } \\
& l_{\text {total }} \quad \text { The total line length }
\end{aligned}
$$

The simulation and result are made up of two types of connection, the first, one power supply and, and the second power supply and seconde double power supply. 


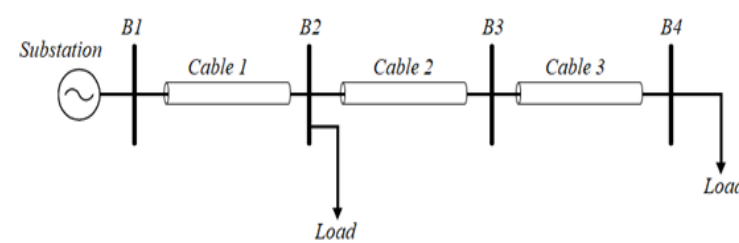

(a)

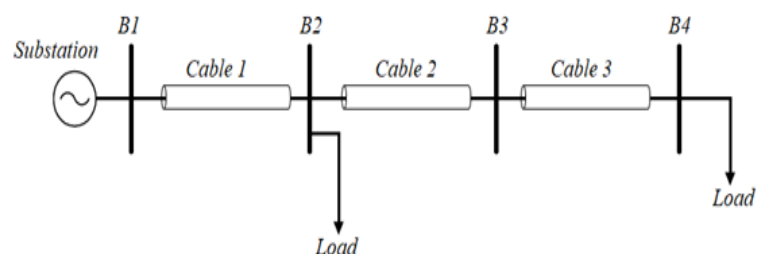

(b)

Figure 4. Distribution network simulation system, (a) single power supply, (b) Double power supply

\subsection{A single power supply}

Figure 5 shows a cable modele to be simulation during the fault in the case of power distribution networks connected to a single power supply. The graphs a-e shown in Figure 6 present the errors in the fault distance of five possible faults as shown in Figures 6 (a)-(e) in the case of a single supply. From Figure 6, it can be seen that the average error obtained using the proposed fault location algorithm is 0.07 for double line-fault and the minimum error value is showed in the case of line to ground fault (0.0001). Therefore, errors obtained using the new fault location algorithm is significantly tolerable for one power supply system, this can prove that the proposed algorithm is suitable for fault location in EPDS.

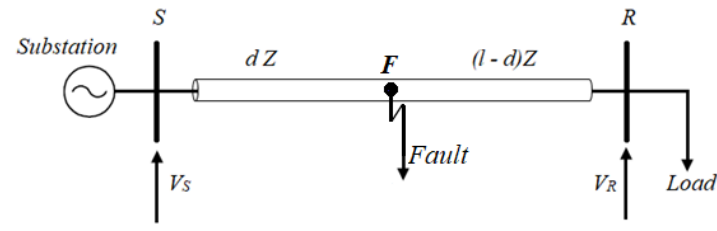

Figure 5. Model of line at a fault in the case of a single power supply

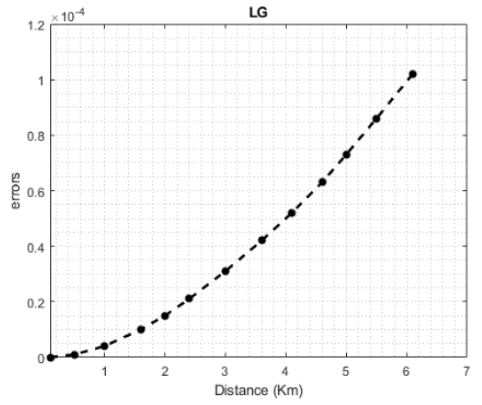

(a)

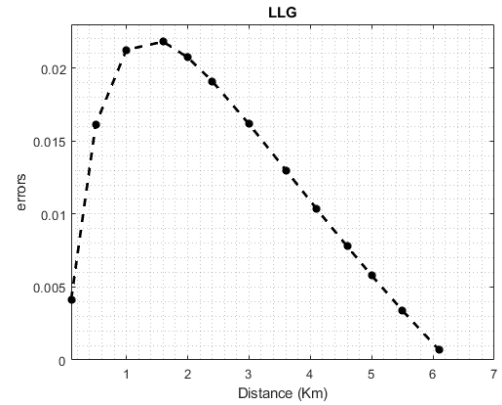

(b)

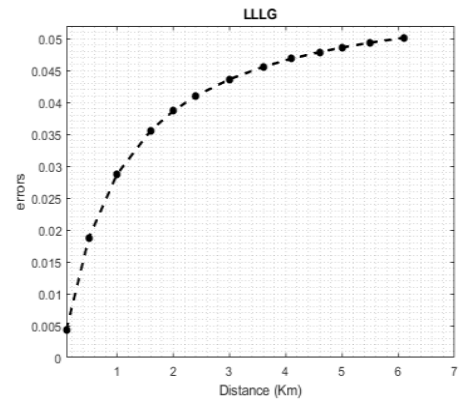

(c)

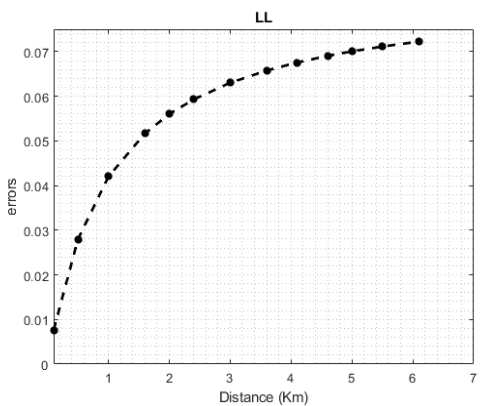

(d)

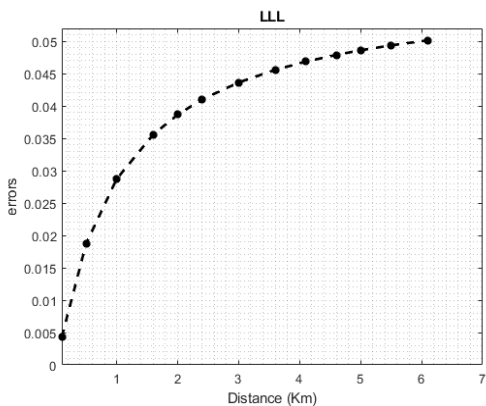

(e)

Figure 6. Result of sumilation for one power supply, (a) line to ground faults (LG), (b) double line to ground faults (LLG), (c) three line to ground faults (LLLG), (d) double-line to fault (LL) and (e) three-line to faults (LLL) 


\subsection{Double power supply}

Figure 7 shows a cable modele to be simulation during the fault in the case of power distribution networks connected to a double power supply. The graphs shown in Figures 8 (a)-(e) present the errors in the fault distance of five possible faults: line-to-ground (LG), double-line-to-ground (LLG), three lines to earth (LLLG), double-line (LL) and three-lines (LLL) in the case of double power supply. From the figure 8, the results of our simulation, we have found a minimum error value (0.02) in the double-phase-to-ground, and the average error is 0.07 for double-line-fault.

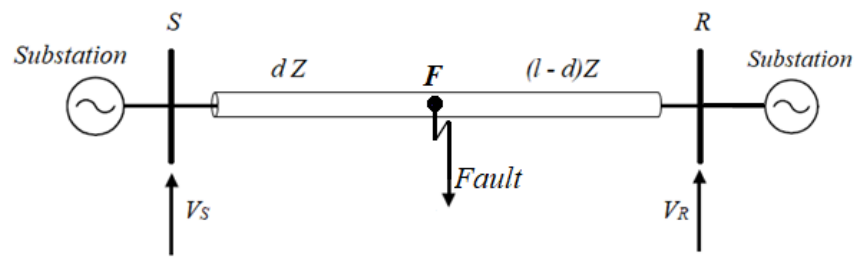

Figure 7. Model of line at a fault in the case of a double power supply

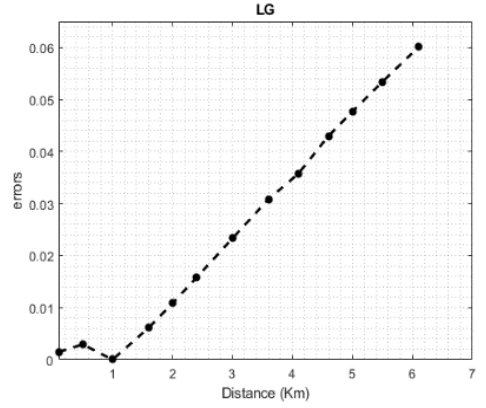

(a)

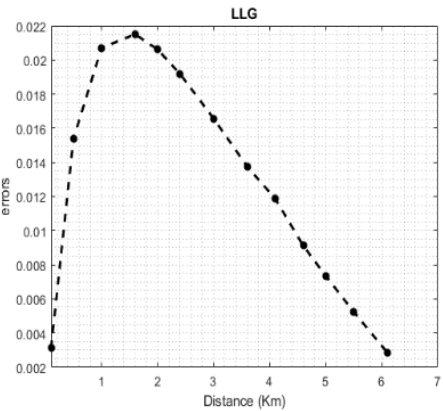

(b)

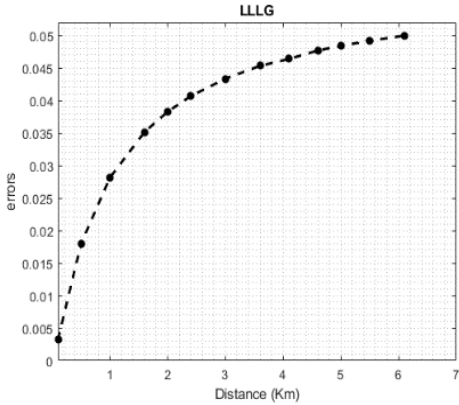

(c)

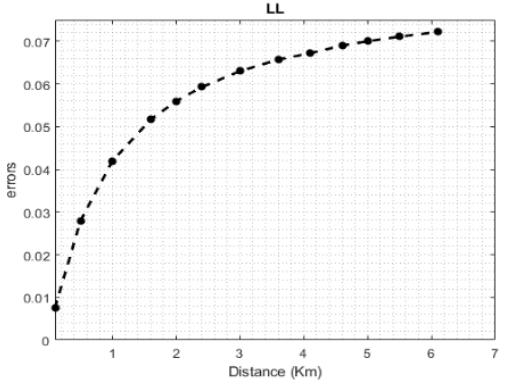

(d)

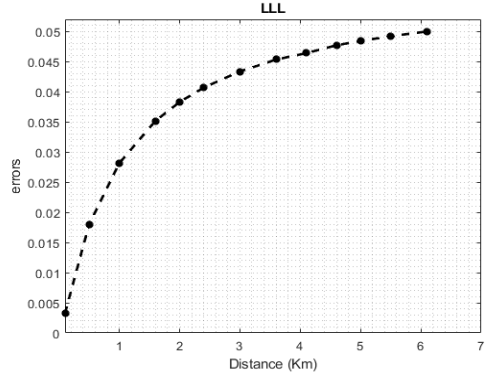

(e)

Figure 8. Result of sumilation for one power supply, (a) Line-to-ground faults (LG), (b) Double-line-toground faults (LLG), (c) Three-line-to-ground faults (LLLG), (d) Double-line fault (LL) and (e) Three-line faults (LLL)

\subsection{Comparison}

The results of a comparison of a smart algorithm and a proposed algorithm by Hing, et al. [26] are shown in Table 1 to examine if a smart algorithm is good for fault location. Table 1 shows that for the three alternative fault cases of single-phase-to-ground, phase-to-ground, and tree-phase-to-ground, the error achieved by a smart algorithm is less than the error obtained by the proposed algorithm. As a consequence, the errors obtained using a smart algorithm show that the suggested approach is capable of locating faults in EPDS. 
Table 1. Comparison of results

\begin{tabular}{ccccc}
\hline \multirow{2}{*}{ Fault Type } & \multicolumn{2}{c}{ A smart algorithm } & \multicolumn{2}{c}{ [26] } \\
& Min Error & Max Error & Min Error & Max Error \\
\hline LG & 0 & 0.0001 & 0.03 & 0.62 \\
LLG & 0.0006 & 0.02 & 0.02 & 0.11 \\
LLLG & 0.004 & 0.05 & 0.01 & 0.1 \\
\hline
\end{tabular}

\section{CONCLUSION}

This paper proposes an impedance based on an algorithme for fault location with precision in power distribution networks (PDN) for both options the one power source and double power source with a high accuracy. This algorithme was developed for all types of faults in distribution systems that is not the case in the current state of the art fault location formulation. The implementation of the proposed method can help energy companies reduce the system restoration time improving their power quality indexes.

\section{REFERENCES}

[1] MM. Saha MM, J. Izykowski, E. Rosolowski, Fault Location on Power Network, Springer-Verlag: London, England, 2010.

[2] S.S. Gururajapathy, H. Mokhlis, and H.A. Illias, "Fault location and detection techniques in power distribution systems with distributed generation: A review," Renewable and Sustainable Energy Reviews, vol. 74, pp. 949-958, 2017, doi: 10.1016/j.rser.2017.03.021

[3] R. Dashti, M. Ghasemi, and M. Daisy, "Fault location in power distribution network with presence of distributed generation resources using impedance-based method and applying $\pi$ line model," Energy, vol. 159, pp. 344-360, 2018, doi: 10.1016/j.energy.2018.06.111.

[4] K. Wang, J. Wang, C. Chang, J. Zhang, L. Jia, and J. Zhao, "A fault location method for multi-branch distribution lines," 2018 14th International Conference on Natural Computation, Fuzzy Systems and Knowledge Discovery (ICNC-FSKD). IEEE, 2018, doi: 10.1109/FSKD.2018.8686870.

[5] A. Saber, "New fault location algorithm for four-circuit overhead lines using unsynchronized current measurements," International Journal of Electrical Power \& Energy Systems, vol. 120, 2020, doi: 10.1016/j.ijepes.2020.106037.

[6] J. Mora-Flòrez, J. Meléndez, and G. Carrillo-Caicedo, "Comparison of impedance-based fault location methods for power distribution systems," Electric Power Systems Research, vol. 78, no. 4, pp. 657-666. doi: 10.1016/j.epsr.2007.05.010.

[7] H. Touijer, Y. Menchafou, H. El Markhi, M. Zahri, and M. Habibi, "Faults Detection in EPDS through a BEAGLEBONE Black Card," Indian Journal of Science and Technology, vol. 11, no. 7, pp. 1-5, 2018, doi: 10.17485/ijst/2018/v11i7/118978.

[8] D. Novosel, D. Hart, and J. Myllymaki, "System for locating faults and estimating fault resistance in distribution networks with tapped loads," U.S. Patent 5839093, 1998.

[9] R. Das, "Determining the locations of faults in distribution systems", Doctoral thesis. University of Saskatchewan, Saskatoon, Canada, 1998, 206 p.

[10] L. Yang, and C. Springs. "One terminal fault location system that corrects for fault resistance effects", US Patent number 5,773,980 1998.

[11] R. Das, M.S. Sachdev, and T.S. Sidhu, "A fault locator for radial sub-transmission and distribution lines", in IEEE Power Engineering Society Summer Meeting, Seattle, WA, USA, July 16-20, 2000, doi: 10.1109/PESS.2000.867627.

[12] M. Saha, and E. Rosolowski, "Method and device of fault location for distribution networks", US Patent number $6,483,4352002$

[13] M.S. Choi, S. Lee, D. Lee, and B. Jin, "A new fault location algorithm using direct circuit analysis for distribution systems”, IEEE Trans. Power Syst. Vol. 9, no. 1, pp. 35-41, 2004, doi: 10.1109/TPWRD.2003.820433.

[14] E. Senger, J. Manassero, G., C. Goldemberg, and E. Pellini, "Automated fault location system for primary distribution networks," IEEE Trans. Power Del., vol. 20, no. 2, pt. 2, pp. 1332-1340, Apr. 2005, doi: 10.1109/TPWRD.2004.834871.

[15] J. Kim, M. Baran, and G. Lampley, "Estimation of fault location on distribution feeders using PQ monitoring data," in IEEE Power Engineering Society General Meeting, Tampa, FL, 2007, doi: 10.1109/PES.2007.385635.

[16] M.C. d'Almeida, F.F. Costa, S. Xavier-de-Souza, and F. Santana, "Optimal placement of faulted circuit indicators in power distribution systems," Electr. Power Syst Res., vol. 81, no. 2, pp. 699-706, 2011, doi: 10.1016/j.epsr.2010.10.037.

[17] R. Krishnathevar, and E.E Ngu, "Generalized impedance-based fault location for distribution systems," IEEE Transactions on Power Delivery, vol. 27 no. 1, pp. 449-451, 2012, doi: 10.1109/TPWRD.2011.2170773.

[18] M. Avendano-Mora, and J.V. Milanovic, "Generalized formulation of the optimal monitor placement problem for fault location,” Electr. Power Syst. Res., vol. 93, pp. 120-126, 2012, doi: 10.1016/j.epsr.2012.07.010.

[19] J. Sadeh, E. Bakhshizadeh, and R. Kazemzadeh, "A new fault location algorithm for radial distribution systems using modal analysis," Int. J. Electr. Power Energ. Syst., vol. 45, pp. 271-278, 2014, doi: 10.1016/j.ijepes.2012.08.053 
[20] X. Wanjing, and L. Yuan, "Novel fault location methods for ungrounded radial distribution systems using measurements at substation," Electric Power Systems Research, vol. 106, pp. 95-100, 2014, doi: 10.1016/j.epsr.2013.08.012.

[21] E. Khoudry, A. Belfqih, T. Ouaderhman, J. Boukherouaa, and F. Elmariami, "A real-time fault diagnosis system for high-speed power system protection based on machine learning algorithms," International Journal of Electrical and Computer Engineering (IJECE), vol. 10, no. 6, pp. 6122-6138, 2020, doi: 10.11591/ijece. v10i6.pp6122-6138.

[22] A. K. Abbas, S. Hamad, and N. A. Hamad, "Single line to ground fault detection and location in medium voltage distribution system network based on neural network," Indonesian Journal of Electrical Engineering and Computer Science, vol. 23, no. 2, pp. 621-632, 2021, doi: 10.11591/ijeecs. v23.i2. pp621-632.

[23] C. G. Arsoniadis, C. A. Apostolopoulos, P. S. Georgilakis, and V, C. Nikolaidis, "A voltage-based fault location algorithm for medium voltage active distribution systems," Electric Power Systems Research, vol. 196, 2021, doi: 10.1016/j.epsr.2021.107236.

[24] J. D. Glover, and S. Sarma M, Power System Analysis and Design, 1994, PWS Publishing Company, Boston.

[25] "Matlab Help Manual," The Mathworks Inc., 2017 b.

[26] T. P. Hinge, and S. S. Dambhare, "Novel fault location algorithm for transmission line using synchronized measurements," In 2016 IEEE/PES Transmission and Distribution Conference and Exposition (T\&D) (pp. 1-6). IEEE, doi: 10.1109/TDC.2016.7519874.

\section{BIOGRAPHIES OF AUTHORS}
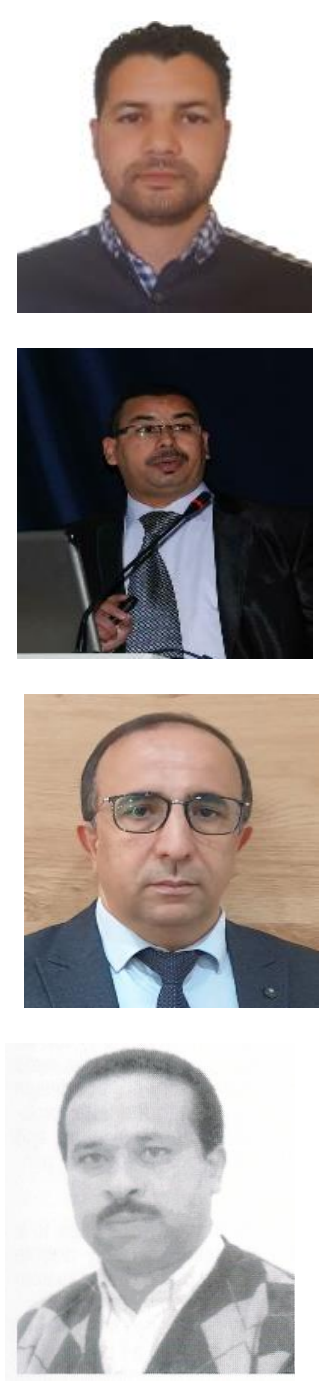

Hamid Touijer was born in Jorf El Melha, Morocco, on 17th August 1987. He received the Master degree in Micro-electronic from Faculty of Science Ibn Tofail University, kenitra - Morocco, in July 2015. He's a PhD student at Laboratory of Electronic Systems, Information Processing, Mechanics and Energetics of University Ibn Tofail Kenitra, Morocco. Email: hamid.touijer@gmail.com

Mohammed el Alami was born in Errachidia, Morocco, on 20th August 1985. He received the Master degree in Telecommunication from National School of Applied Sciences ENSA of University of Sidi Mohammed ben Abdellah Fez, in July 2011. He received his Doctorate degree in Electric Engineering from the Faculty of Science at Laboratory of Electronic Systems, Information Processing, Mechanics and Energetics of University Ibn Tofail Kenitra, Morocco. Email: elalami.ensa@gmail.com_

Mustapha Zahri was born in Agadir Morocco on August 1, 1969. He received the electrical engineering degree in 1993 from superior national school from electricity and mechanics (ENSEM) Casablanca Morroco. In 2017, He received his Doctorate degree in Electric engineering from the faculty of science at Laboratory of Electronic Systems, Information Processing, Mechanics and Energetics of university Ibn Tofail Kenitra Morocco. Since 9 marche 2021, he is General Manager of the Electricity and Water Distribution company in El Jadida Morocco. Email:_mustapha.zahri@gmail.com

Mohamed Habibi was born in 1957 in Khemisset in Morocco. He received his Thesis of University of 3 Cycle (Electronics) from the University of Sciences and Techniques, Lille Flandres Artois, France, in 1985 and the State doctoral thesis (Electronics) from Engineer School Mohammedia of the University Mohamed V, Rabat, Morocco, in 1993. He was a member of (the Laboratoire) the Laboratory of e electronic and Communications since 1989 at the 'Engineer School Mohammedia', Rabat. He was responsible of the Automatic Laboratory and microwave (LAMO) and presently he is a member of the Laboratory of Electronic Systems, Information Processing, Mechanics and Energetics. He is a Professor of Electrical Engineering at the University lbn Tofail, Faculty of Science, Department of Physique, Kenitra, Morocco since 1985. He is working on applications of Microwaves. Email: habibi.mohamed@uit.ac.ma 